\title{
Characterization of a Novel HIV-1 Recombinant Form (CRF01_AE/CRF07_BC/CRF08_BC) Identified from Guangxi, China
}

\author{
Bingyu Liang, ${ }^{1, *}$ Yao Yang, ${ }^{1, *}$ Fei Zhang, ${ }^{1}$ Kang Li, ${ }^{1}$ Yuan Yang, ${ }^{2}$ Weidong Ou, Jiegang Huang, \\ Chuanyi Ning, ${ }^{1,2} \mathrm{Li} \mathrm{Ye}^{1,2}$ and Hao Liang ${ }^{1,2}$
}

\begin{abstract}
Recently, more and more kinds of circulating recombinant forms (CRFs) and unique recombinant forms (URFs) were identified in the population in China. A novel HIV-1 recombinant virus (2016GXNNIDU016) was identified in this study, which was isolated from an HIV-1 infected intravenous drug user in Nanning, the capital of Guangxi, China. Phylogenetic analysis of the near full-length genome (NFLG) indicated that the sequence (2016GXNNIDU016) was a monophyletic branch that did not cluster with any previously identified genotype. Recombinant analysis showed that the NFLG of 2016GXNNIDU016 is composed of CRF07_BC, CRF01_AE, and CRF08_BC, including nine mosaic segments. Differing from previously documented CRF01_AE and CRF07/08_BC recombinant forms, 2016GXNNIDU016 increases the genetic complexity of HIV-1 in Guangxi. The constant emergence of novel recombinant forms should draw our attention to make more efforts in supervising and preventing the spread of HIV infection.
\end{abstract}

Keywords: HIV-1, recombinant form, CRF01_AE/CRF07_BC/CRF08_BC, URF

$\mathbf{H}$ UMAN IMMUNODEFICIENCY VIRUS type 1 (HIV-1) is characterized by high variability due to the high mutation and recombination rates of reverse transcriptase during the process of viral replication. ${ }^{1,2}$ Multiple HIV strains coepidemic in the same region have provided the opportunity for the emergence of new recombinants. A new circulating recombinant form (CRF) of HIV is identified when the new recombinant forms have been isolated from more than three epidemiologically unlinked subjects. Similarly, unique recombinant forms (URFs) refer to new recombinants found in only one or two individual patients. According to the most recent records in the Los Alamos National Laboratory HIV sequence database, at least 98 strains of CRFs have been recorded worldwide (https://www.hiv.lanl.gov/content/ sequence/HIV/CRFs/CRFs.html).

Guangxi province is located in southwest China. HIV transmission in Guangxi was primarily fuelled initially by intravenous drug use due to its location along a major heroin trafficking route linking Guangxi with Yunnan and Vietnam and its close proximity to the world's major heroin-producing area, known as the Golden Triangle. ${ }^{3}$ Former research has identified CRF01_AE, CRF07_BC, and CRF08_BC as the three major genotypes in Guangxi. ${ }^{4}$ CRF_01AE was first identified in female sex workers who returned from Thailand in Yunnan, ${ }^{5,6}$ which prevailed in injecting drug users (IDUs) in the late 1980s and then shifted to heterosexuals in the early 1990 s. $^{7}$ In addition, CRF07_BC/CRF08_BC was first transited in Guangxi from Yunnan IDUs in 1994 and 1997, respectively. ${ }^{8,9}$ CRF08_BC was the major circulating genotype in IDUs, ${ }^{10}$ and CRF07_BC was responsible for a significant portion of infection among men who had sex with men and heterosexuals. Co-epidemics of multiple HIV strains led to Guangxi as a hotbed for generating the new CRFs and URFs. ${ }^{11}$ It was reported that new recombinant forms between CRF_01AE and CRF_07/08_BC have emerged in Guangxi, ${ }^{2, \overline{12}-14}$ but none of the novel genotypes contains more than three CRFs in Guangxi. In this present study, a novel recombinant form was isolated from an IDU in Nanning, the capital of Guangxi Zhuang autonomous region. This demonstrates the constant emergent new HIV-1 genotypes under current cocirculation of multiple genotype circumstances. $^{15}$

\footnotetext{
${ }^{1}$ Guangxi Key Laboratory of AIDS Prevention and Treatment \& Guangxi Universities Key Laboratory of Prevention and Control of Highly Prevalent Disease, School of Public Health, Guangxi Medical University, Nanning, China.

${ }^{2}$ Guangxi Collaborative Innovation Center for Biomedicine, Life Science Institute, Guangxi Medical University, Nanning, China.

*These authors contributed equally to this work.
} 
This new sequence was identified in a sample from an intravenous drug user in Nanning, a married 43-year-old female, unemployed with a junior diploma. The proviral DNA was isolated from the patient's peripheral blood lymphocyte using AIDLAB DNA FIT according to the manufacturer's instructions and amplified using nested polymerase chain reaction with the same cycling conditions in both rounds: Preheat at $94^{\circ} \mathrm{C}$ for $2 \mathrm{~min}$, followed by denaturation for 35 cycles at $94^{\circ} \mathrm{C}$ for $15 \mathrm{~s}$, at $60^{\circ} \mathrm{C}$ for $30 \mathrm{~s}$, at $68^{\circ} \mathrm{C}$ for $4 \mathrm{~min}$, a final extension at $68^{\circ} \mathrm{C}$ for $10 \mathrm{~min}$, and preserved at $8^{\circ} \mathrm{C}$. Then the sample was sent to Tianyi Huiyuan Bioscience \& Technology Inc. for purification and sequencing. After acquiring a near full-length genome (NFLG) of $8,981 \mathrm{bp}$, the 2016GXNNI DU016 sequence was first submitted to the online quality control tool (https://www.hiv.lanl.gov/content/sequence/QC/ index.html) to exclude possible assembling mistake.

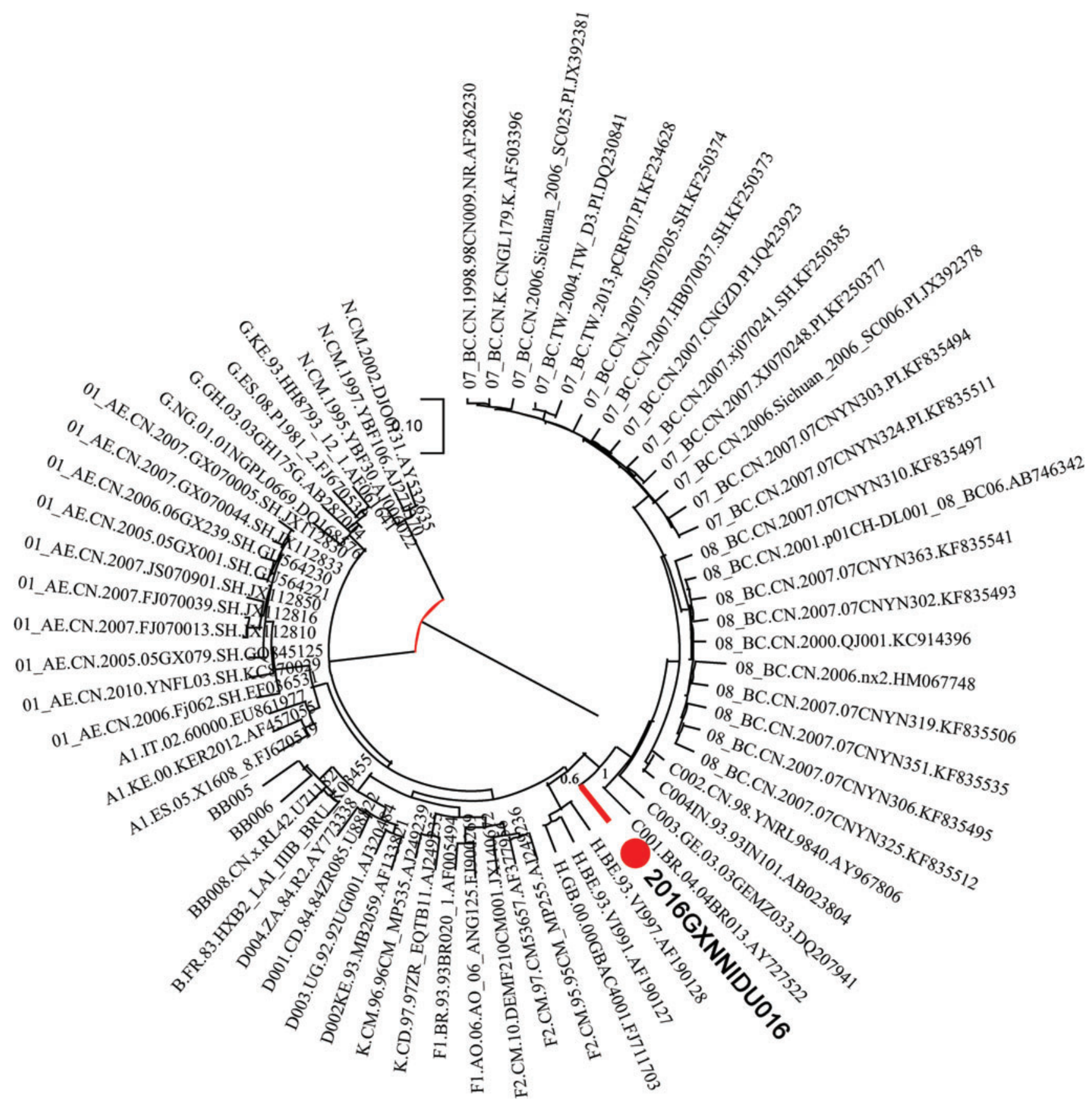

FIG 1. Phylogenetic tree analyses of the NFLG sequence of 2016GXNNIDU016. The tree was constructed by FastTree in external command of AliView, using approximately maximum likelihood method with the GTR model. 2016GXNNIDU016 clustered with no one reference sequences and forming a monophyletic branch supported by a high bootstrap value of 1. 2016GXNNIDU016 was marked by the solid circle (O) and bold line. Bootstrap values greater than 0.7 would be considered stable in the phylogenetic tree. GTR, general time reversible; NFLG, near full-length genome. Color images are available online. 
To further determine whether the 2016GXNNIDU016 sequence represented a new recombinant form, we downloaded the reference sequences from the Los Alamos HIV Sequences Database (https://www.hiv.lanl.gov/content/ sequence/HIV/mainpage.html) and aligned them using AliView V 1.25. The NFLG phylogenetic tree was constructed by the FastTree V2.1 based on the approximately maximum likelihood method with the general time reversible (GTR) model and adjusted with Figtree V1.4.3. Bootstrap values greater than 0.7 were considered stable (Fig. 1).

The online tool "Recombinant Identification Program" (RIP; https://www.hiv.lanl.gov/content/sequence/RIP/RIP .html) was then used to identify the basic constituents of this sequence. We utilized the online tool "jumping profile Hidden Markov Model" (jpHMM; http://jphmm.gobics.de) and Bootscan with SimPlot V3.5.1 to perform preliminary recombinant analysis and breakpoint determination. According to the results of Bootscan (Fig. 2A) and jpHMM, there were eight breakpoints dividing the sequence 2016GXNNIDU016 into nine segments, including subtype C and CRF01_AE, listed

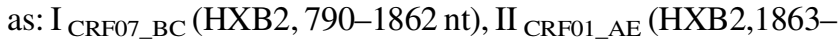
$2547 \mathrm{nt})$, III CRF07_BC (HXB2, 2548-3094 nt), IV CRF01_AE (HXB2, 3095-5556 nt), V CRF07_BC (HXB2, 5557-5920 nt), VI CRF01_AE (HXB2, 5921-6326 nt), VII CRF08_BC (HXB2, 6327-8307 nt), VIII CRF01_AE (HXB2, 8308-9121 nt), and IX CRF08_BC (HXB2, 9122-9412 nt). However, considering that the prevalence of the pure subtype $\mathrm{C}$ is much less than the recombinant subtype CRF07/08_BC in Guangxi, it is possible that the location where the $\mathrm{C}$ segment is inserted may come from the CRF07_BC and CRF08_BC instead of the pure $\mathrm{C}$ subtype. Next, the nearest reference sequences of each segment were chosen to construct the subgenome phylogenetic tree according to the same means mentioned above, but more CRF07_BC, CRF08_BC, and CRF01_AE were added. All the phylogenetic trees were embellished with MEGA $X$ and Adobe Photoshop Creative Cloud 2018. After confirming the

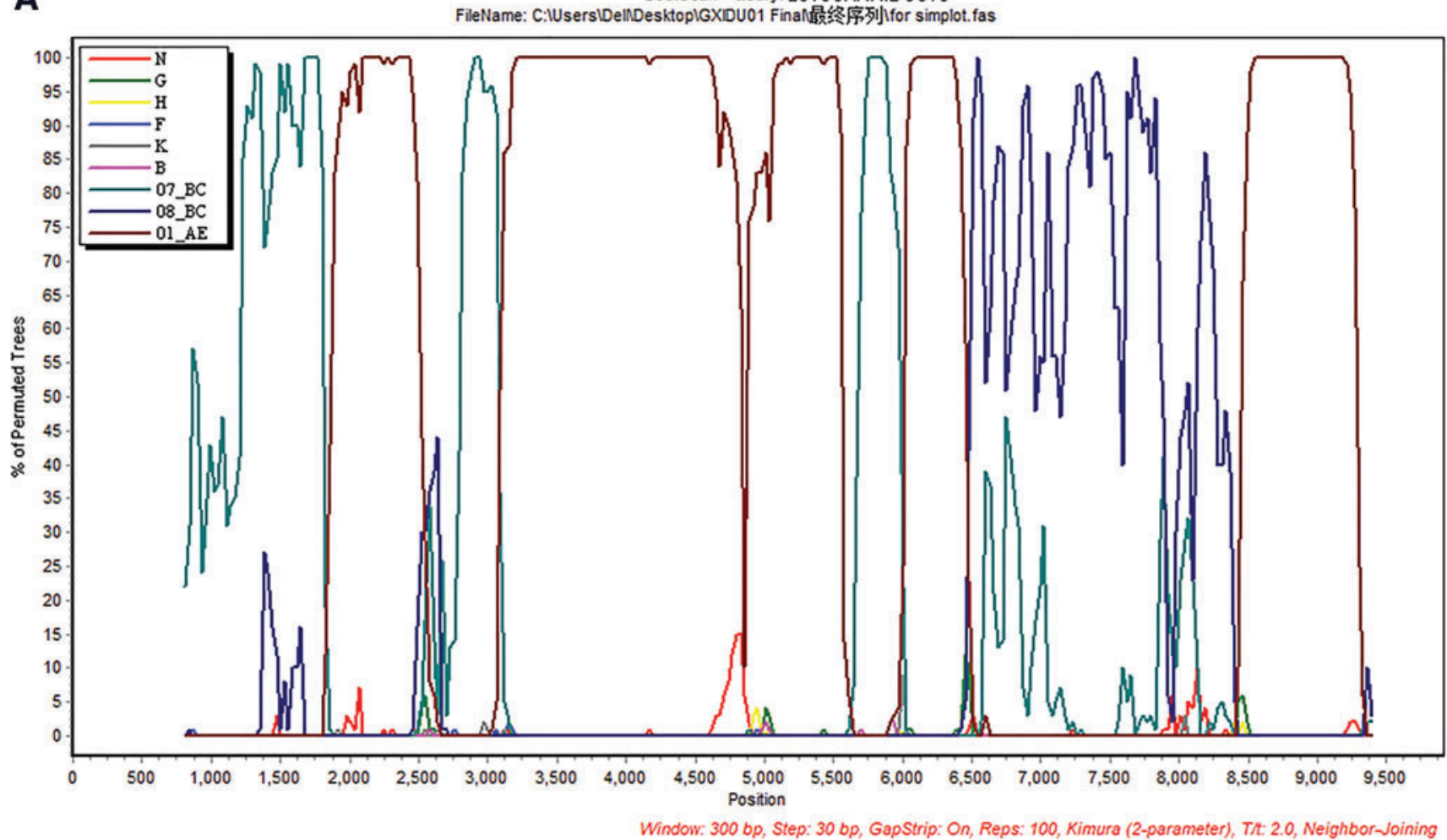

B

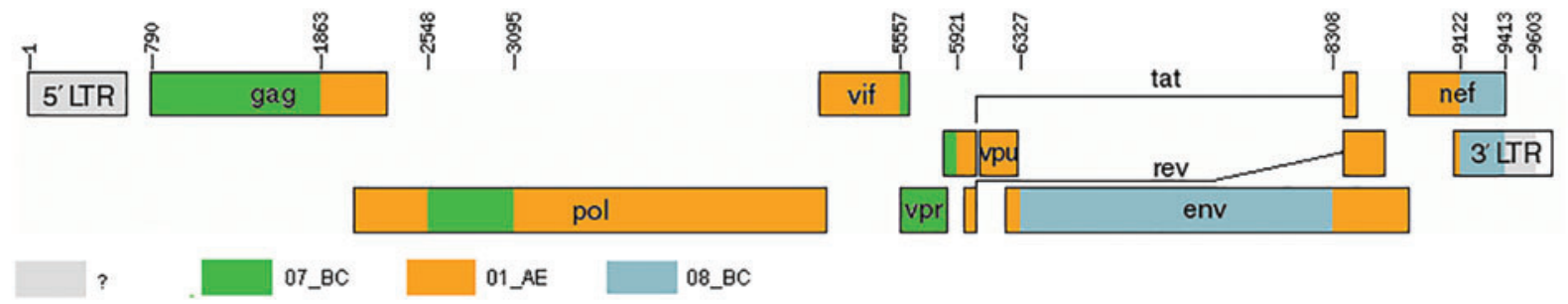

FIG 2. Bootscan results and recombinant mosaic model of the 2016GXNNIDU016 near full-length genome. (A) Bootscan plots of 2016GXNNIDU016 using CRF08_BC, CRF01_AE, CRF07BC, and common subtype B, F1, K, H, G, N as references. 1, 7, 8 represent CRF 01/07/08, respectively, and 01 represents 2016GXNNIDU037. The parameters of Bootscan analysis were $300 \mathrm{bp}$ on Window and $30 \mathrm{bp}$ on step. (B) The genome map of the NFLG sequence of 2016GXNNIDU016 was generated using the Online Recombinant HIV-1 Drawing Tool (https://www.hiv.lanl.gov/content/ sequence/DRAW_CRF/recom_mapper.html). Eight breakpoints divided the NFLG into nine unique segments. CRF, circulating recombinant form. Color images are available online. 

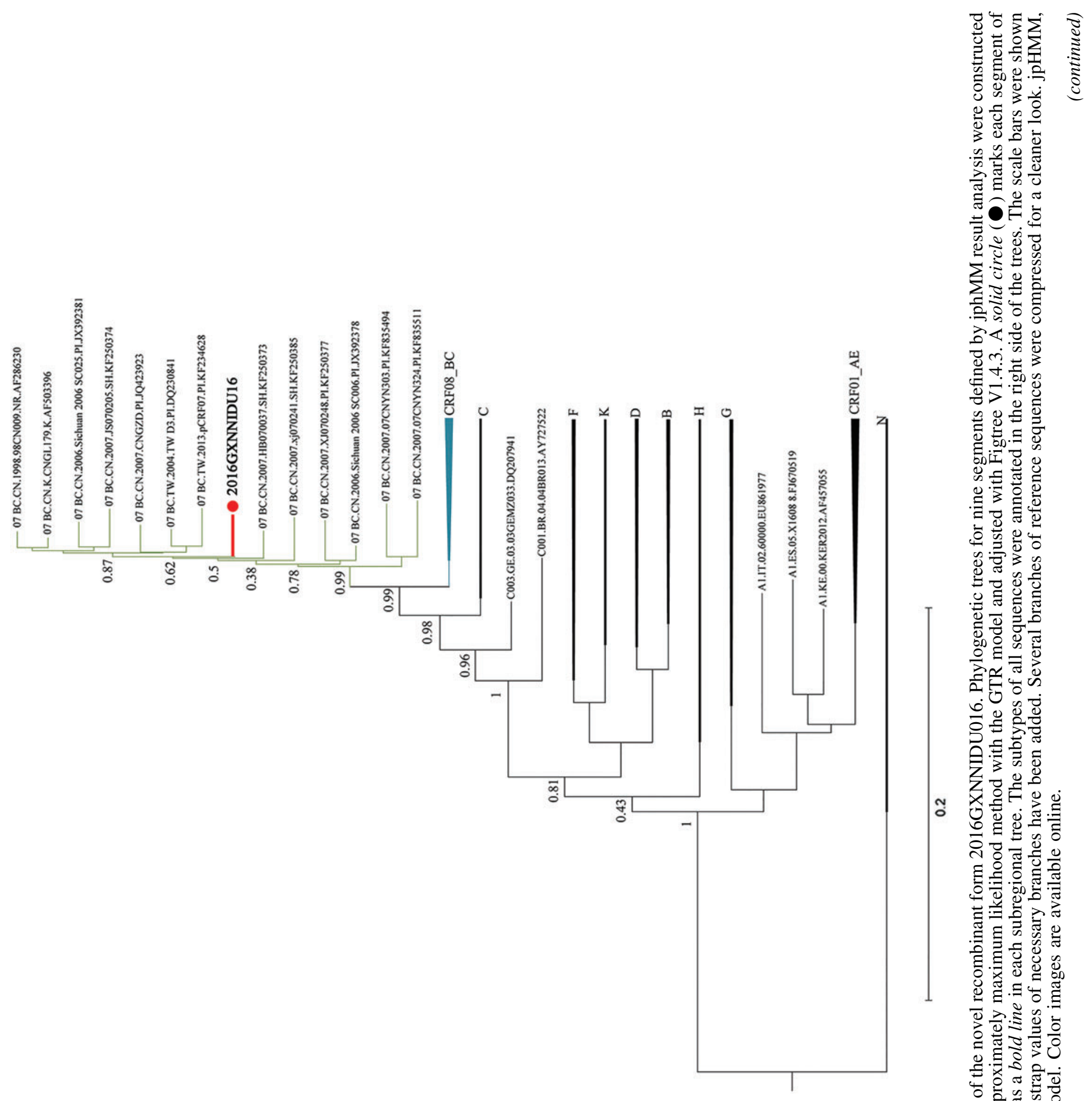

过灾

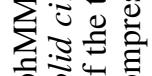

득 눙 它《造

更的

牙方:

节氖

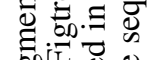

क. जٓ

过

驱

훈둥

诃

월 \&

会言䎡

乙过

กุ

동

约

귱픈웡

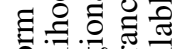

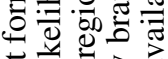

畨

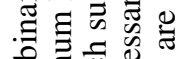

हี. छ

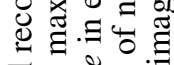

ए

콩

武通

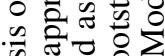

可司完?

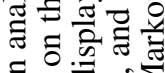

oี

6.

苛 은

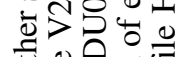

害递貝氞

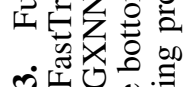

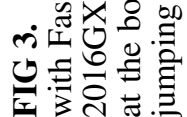



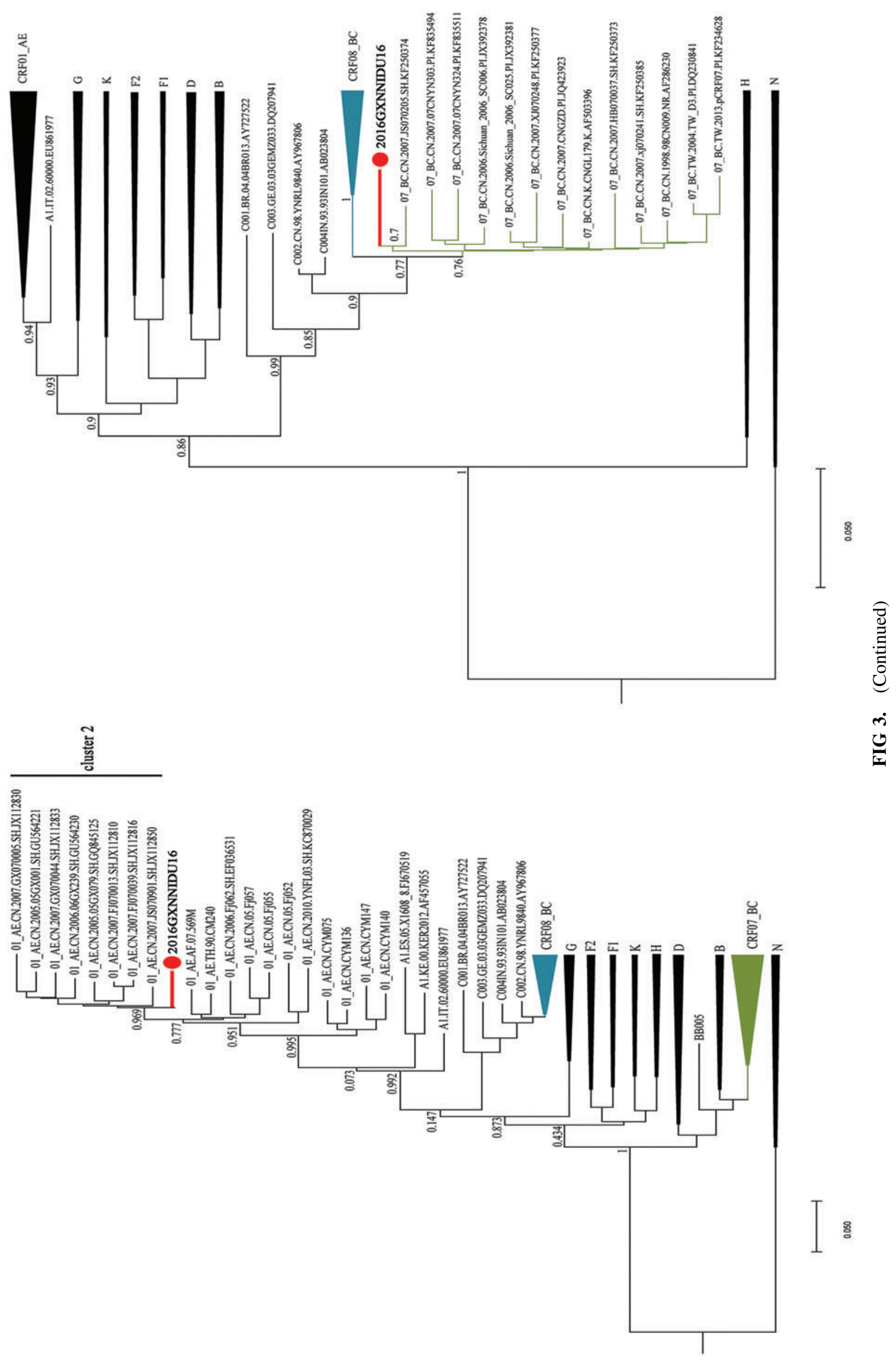

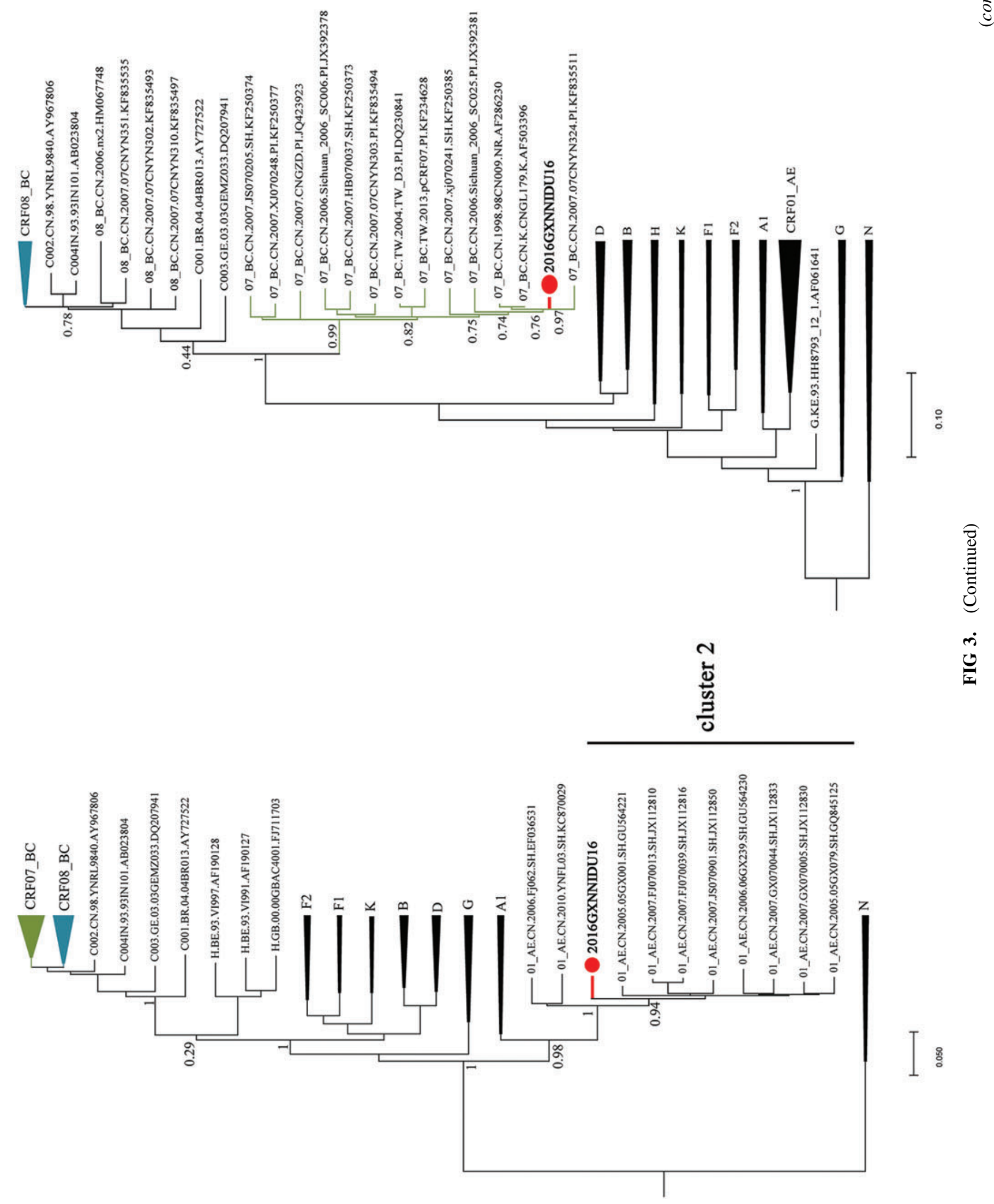


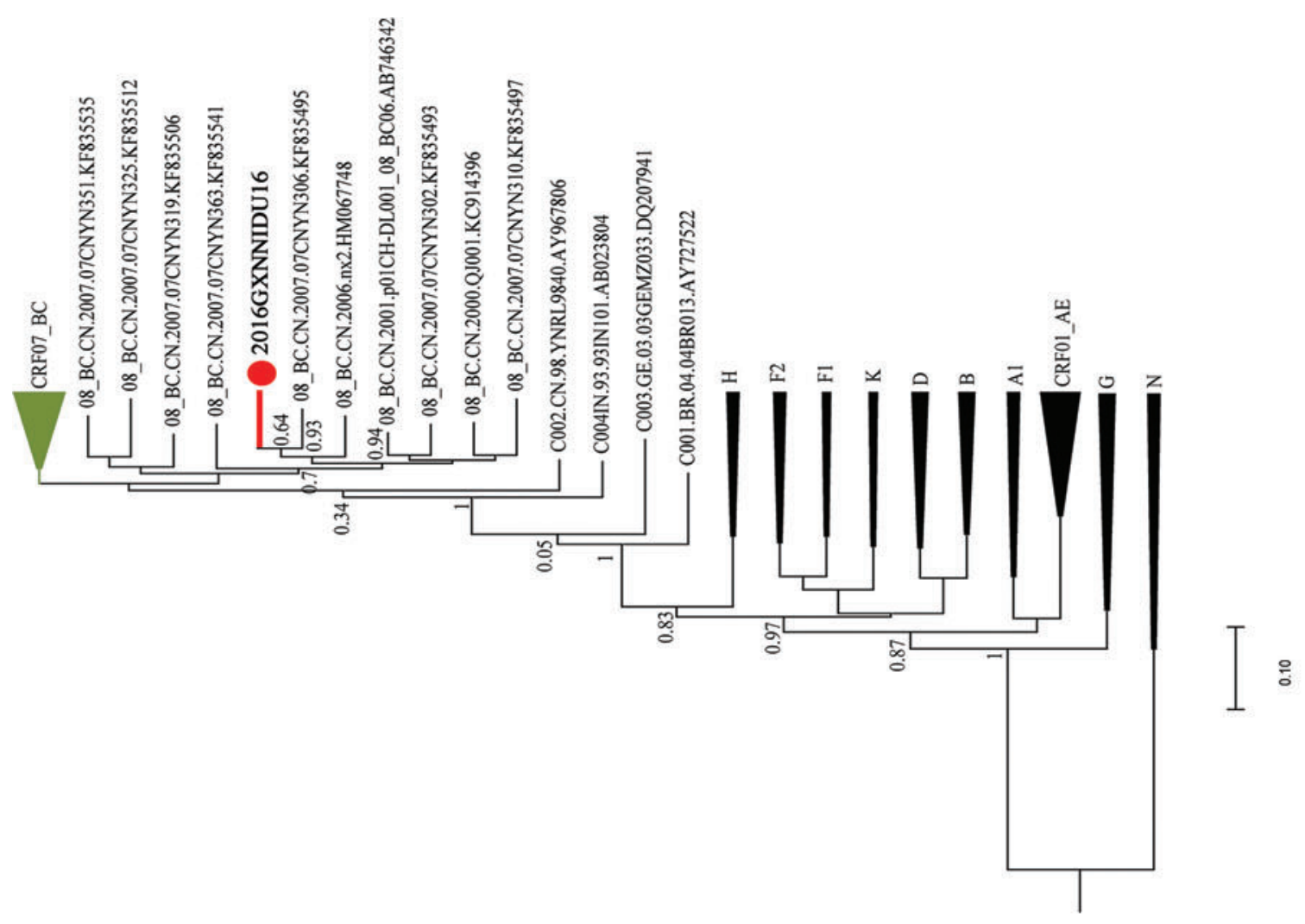

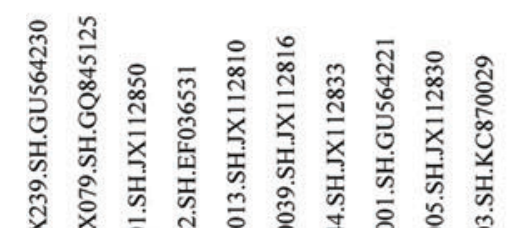

त्रे

荕

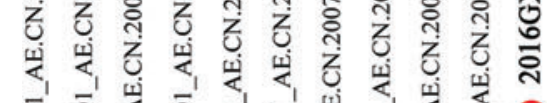

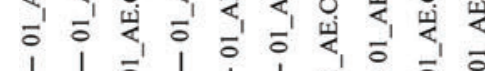

$\bigsqcup \quad j \quad$ L

ڤ.

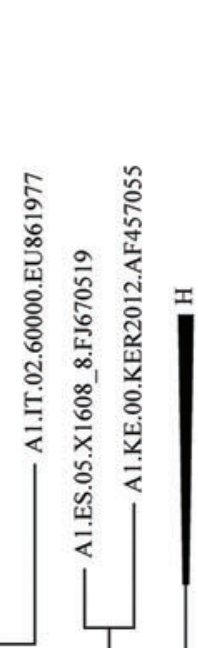

ॠ

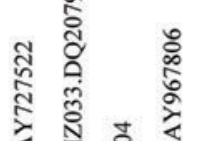

离

$\dot{0}$

เุํ․

के

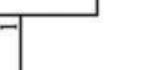

n

可!

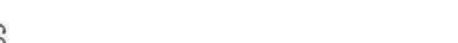



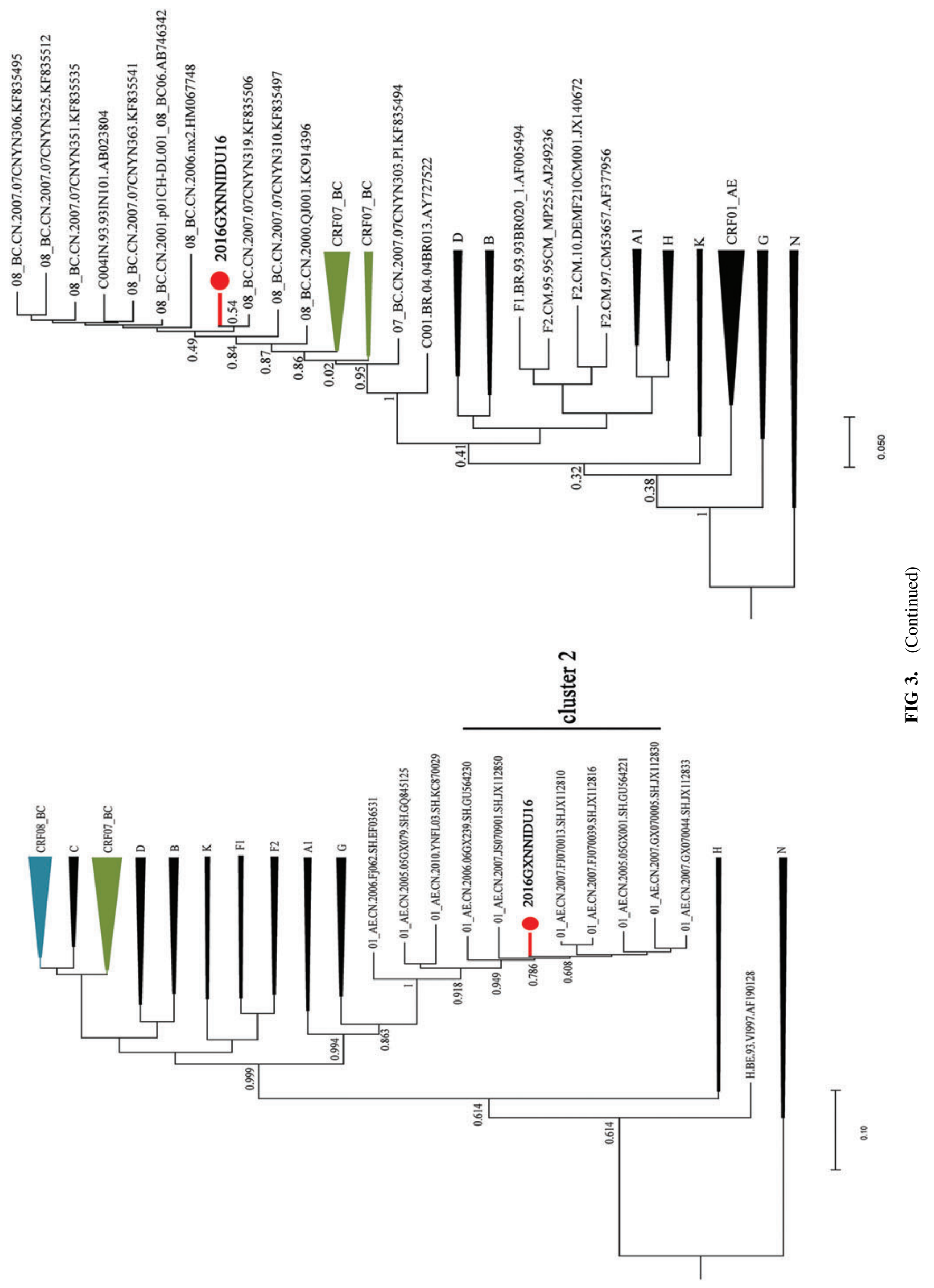
recombinant structure of 2016GXNNIDU016, the recombinant map was drawn with the Online Recombinant HIV-1 Drawing Tool (https://www.hiv.lanl.gov/content/sequence/DRAW_ CRF/recom_mapper.html).

Figure 1 indicates that 2016GXNNIDU016 forms a monophyletic cluster with pretty high stability (bootstrap value $=1$ ), which did not belong to any existing subtype clusters. That is, 2016GXNNIDU016 is a brand new recombinant form of the HIV-1 genotype. The subgenome phylogenetic tree further confirmed the recombinant structure of: I CRF07_BC $(\mathrm{HXB} 2,790-1862$ nt), II CRF01_AE $(\mathrm{HXB} 2$, 1863-2547 nt), III CRF07_BC (HXB2, 2548-3094 nt), IV CRF01_AE (HXB2, 3095-5556 nt), V CRF07_BC (HXB2, 5557$5920 \mathrm{nt}), \mathrm{VI}$ CRF01_AE (HXB2, 5921-6326 nt), VII CRF08_BC (HXB2, 6327-8307 nt), VIII CRF01_AE (HXB2, 8308-9121 nt), and IX CRF08_BC (HXB2, 9122-9412 nt) (Fig. 3). It is complicated enough with no main subtype as a backbone but collaged with three different CRFs in this sequence. The longest segment belongs to the env gene, a $1,980 \mathrm{bp}$ of CRF_08BC, the second-longest segment is a length of CRF_01AE in the pol gene, and the longest segment in the gag gene is CRF_07BC. The derived recombinant map provides a visualization of these results (Fig. 2B).

According to former reports, several CRF_01AE strains identified in Guangxi in the late 90s were closely related to the prevalent strains carried by IDUs of northern Vietnam. ${ }^{16}$ Segment II, IV, and VIII gather with the CRF_01AE cluster 2 , which includes IDUs and heterosexuals as transmission conduits prevailing in Guangxi, Fujian, Jiangsu, and North Vietnam based upon the criterion illustrated by Feng et al. ${ }^{17}$ The nearest branches of this sequence are all heterosexually transmitted, which contributes to an HIV-infection shift from a high-risk group to the general population. Since the domain transmission route transferred from blood exchange to sexual intercourse, CRF01_AE has become the main strain responsible for HIV-1 infection in mainland China. ${ }^{18}$ The subject in our study is a middle-aged, of Han nationality, junior diploma, and jobless unmarried female, who was infected by sharing needles. According to another study, individuals that were jobless, belonged to a minor ethnicity, had a low education, and lived in a bordering city had a tendency toward needle-sharing behavior. However, needle-sharing behaviors were mainly due to drug-use behaviors rather than sociodemographic factors. ${ }^{19}$ It is imperative for the department concerned to control the drug smuggling business and to promote antidrug education to reduce HIV-1 infection through needle sharing.

In addition, referencing the retrieval results of PubMed, five recombinant forms have been identified in Guangxi in recent years, including one recombinant of CRF01_AE/B/C, ${ }^{14}$ one recombinant of CRF01_AE/CRF08_BC, ${ }^{20}$ two recombinants of CRF01_AE/CRF07_BC, ${ }^{2,12}$ and a recombinant of CRF01_AE/CRF07_BC/B. ${ }^{13}$ Recombination commonly occurs between CRF01_AE and CRF07_BC. However, there were a few recombinant forms containing more than two CRFs that seldom recombined with CRF08_BC. Our study provides a unique genotype consisting tree of CRFs, including CRF08_BC. A high variety of genotypes, together with high rates of viral replication, may limit the intra- and intersubtype cross-reactivity of the immune response. ${ }^{21}$ This means that under the current situation of high genetic variety in Guangxi, the development of vaccines has a tremendous difficulty to make immunogens that match the sequences present in the target population as closely as possible. This may also be a challenge to treatment programs and lead to increased prevalence.

In conclusion, we identified a unique near full-length genomic sequence of the CRF08_BC, CRF_01AE, and CRF_07BC recombinant of HIV-1 isolated from a female IDU in Guangxi, which was distinct from the CRFs and URFs previously reported. Therefore, we should continuously supervise the HIV-1 molecular epidemiology information to gather enough information for vaccine design and provide effective suggestions for prevalence control.

\section{Sequence Data}

The NFLG sequence of 2016GXNNIDU016 has been submitted to GenBank with the accession number MN044632.

\section{Authors' Contributions}

L.Y. and H.L. conceived and designed the study. B.L., Y.Y., and C.N. conducted the data analysis, literature review, and drafted the article. J.H. and Y.Y. were involved in data collection and interpretation. K.L., W.O., and F.Z. assisted with data management and data analysis. All authors contributed to the revision of the article and approved the final version.

\section{Author Disclosure Statement}

No competing financial interests exist.

\section{Funding Information}

This study was supported by the National Key Science and Technology Project of China (grant no. 2018ZX10101002001-006), National Natural Science Foundation of China (grant number 81560326), and Guangxi Natural Science Foundation (2016GXNSFBA380189, 2018GXNSFAA138070).

\section{References}

1. Zhang Y, Pei Z, Li H, et al.: Characterization of a novel HIV-1 circulating recombinant form (CRF80_0107) among men who have sex with men in China. AIDS Res Hum Retroviruses 2019;35:419-423.

2. Kong D, Wang Y, Wang C, et al.: Characterization of a new HIV-1 CRF01_AE/CRF07_BC recombinant virus in Guangxi, China. AIDS Res Hum Retroviruses 2017;33: 1166-1170.

3. Beyrer C, Razak MH, Lisam K, Chen J, Lui W, Yu XF: Overland heroin trafficking routes and HIV-1 spread in south and south-east Asia. AIDS 2000;14:75-83.

4. Li J, Feng Y, Shen Z, et al.: HIV-1 transmissions among recently infected individuals in Southwest China are predominantly derived from circulating local strains. Sci Rep 2018;8:12831.

5. Cheng H, Zhang J, Capizzi J, Young NL, Mastro TD: HIV1 subtype E in Yunnan, China. Lancet 1994;344:953-954.

6. Yu XF, Chen J, Shao Y, Beyrer C, Lai S: Two subtypes of HIV-1 among injection-drug users in southern China. Lancet 1998;351:1250.

7. Liao H, Tee KK, Hase S, et al.: Phylodynamic analysis of the dissemination of HIV-1 CRF01_AE in Vietnam. Virology 2009;391:51-56. 
8. Meng Z, Xin R, Zhong P, et al.: A new migration map of HIV-1 CRF07_BC in China: Analysis of sequences from 12 provinces over a decade. PLoS One 2012;7:e52373.

9. Tee KK, Pybus OG, Li XJ, et al:: Temporal and spatial dynamics of human immunodeficiency virus type 1 circulating recombinant forms 08_BC and 07_BC in Asia. J Virol 2008;82:9206-9215.

10. Li L, Sun G, Liang S, et al.: Different distribution of HIV-1 subtype and drug resistance were found among treatment naive individuals in Henan, Guangxi, and Yunnan province of China. PLoS One 2013;8:e75777.

11. Hemelaar J: The origin and diversity of the HIV-1 pandemic. Trends Mol Med 2012;18:182-192.

12. Dong Z, Shen Z, Xiong R, et al.: Near full-length genomic characterization of a novel HIV type 1 CRF01_AE/CRF07_ $\mathrm{BC}$ recombinant form transmitted between a heterosexual couple in Guangxi, China. AIDS Res Hum Retroviruses 2016;32:689-693.

13. Kong D, Wang Y, Wang C, et al.: Identification of a novel HIV-1 unique recombinant form between B, CRF01_AE and CRF07_BC in men who have sex with men in Guangxi, China. AIDS Res Hum Retroviruses 2018;34:319-323.

14. Wang C, Wang Y, Kong D, Liang S, Feng Y, Ma L: Identification of a novel HIV-1 B/C/CRF01_AE recombinant isolate in Guangxi, China. AIDS Res Hum Retroviruses 2018;34:635-639.

15. Xiao P, Li J, Fu G, Zhou Y, Huan X, Yang H: Geographic distribution and temporal trends of HIV-1 subtypes through heterosexual transmission in China: A systematic review and meta-analysis. Int J Environ Res Public Health 2017; 14:pii: E830 .

16. Kato K, Shiino T, Kusagawa S, et al.: Genetic similarity of HIV type 1 subtype $E$ in a recent outbreak among injecting drug users in northern Vietnam to strains in Guangxi Province of southern China. AIDS Res Hum Retroviruses 1999;15:1157-1168.

17. Feng $\mathrm{Y}, \mathrm{He} \mathrm{X}$, Hsi $\mathrm{JH}$, et al:: The rapidly expanding CRF01_AE epidemic in China is driven by multiple lineages of HIV-1 viruses introduced in the 1990s. AIDS 2013; 27:1793-1802.
18. Wang $\mathrm{X}, \mathrm{He} \mathrm{X}$, Zhong $\mathrm{P}$, et al.: Phylodynamics of major CRF01_AE epidemic clusters circulating in mainland of China. Sci Rep 2017;7:6330.

19. Chen $\mathrm{X}$, Zhu L, Zhou YH, et al.: Factors associated with needle sharing among people who inject drugs in Yunnan, China: A combined network and regression analysis. Infect Dis Poverty 2016;5:73.

20. Wang N, Wei H, Xiong R, et al.: Near full-length genome characterization of a new CRF01_AE/CRF08_BC recombinant transmitted between a heterosexual couple in Guangxi, China. AIDS Res Hum Retroviruses 2014;:484488.

21. Li X, Li W, Zhong P, et al:: Nationwide Trends in Molecular Epidemiology of HIV-1 in China. AIDS Res Hum Retroviruses 2016;32:851-859.

Address correspondence to: Hao Liang Guangxi Collaborative Innovation Center for Biomedicine Life Science Institute Guangxi Medical University Nanning 530021 Guangxi China

E-mail: ianghao@gxmu.edu.cn

$\mathrm{LiYe}$

Guangxi Key Laboratory of AIDS Prevention and Treatment \& Guangxi Universities Key Laboratory of Prevention and Control of Highly Prevalent Disease

School of Public Health

Guangxi Medical University Nanning 530021

Guangxi China

E-mail: yeli@gxmu.edu.cn 